\title{
Dados Abertos Educacionais: Uma Revisão da Literatura Brasileira
}

\author{
Philippe Santos $^{1}$, Rafael Ferreira ${ }^{1}$, Péricles Miranda ${ }^{1}$ \\ ${ }^{1}$ Departamento de Estatística e Informática \\ Universidade Federal Rural de Pernambuco (UFRPE) \\ \{phil.santos13, rafaelflmello, periclesmiranda\}@gmail.com
}

\begin{abstract}
Recently several initiatives to provide open data in different domains, including education, are emerging. Faced on this context, this paper presents an analysis of the main papers using open educational data published in Brazil since 2007. This literature review included scientific articles published in conferences and scientific journals in the field of computer and education, as well as theses and dissertations related to the theme. The results show that the computer and education community in Brazil began to be more interested in this area from 2013. Thus, there are few papers reported. This paper plays an important role because it presents the research dimension in this field, aiming to promote more works.
\end{abstract}

Resumo. Recentemente estão surgindo iniciativas para a disponibilização de dados abertos em várias áreas, entre elas a educação. Diante disto, este trabalho apresenta uma análise dos principais trabalhos que utilizam dados abertos educacionais publicados no Brasil desde o ano de 2007. Esta revisão da literatura abrangeu artigos de eventos científicos e dos periódicos na área de informática e educação, além de teses e dissertações relacionadas com a temática. Os resultados encontrados mostram que a comunidade de informática na educação no Brasil começou a se interessar por esse área a partir de 2013, por isso ainda não existem muitos trabalhos relatados. Por essa razão esse artigo tem fundamental importância para dar a dimensão da pesquisa nessa área, afim de tentar alavancar os trabalhos na mesma.

\section{Introdução}

Com a disseminação da Internet, está cada dia mais fácil disponibilizar conteúdo através de vários meios, por exemplo: redes sociais, fóruns de discussão, vídeos online, emails, entre outros. Contudo, a forma como os dados estão disponibilizados é próprio para consumo humano [Wood et al. 2013]. Em outras palavras, é difícil criar aplicações computacionais que se beneficiem desses dados. Para lidar com esse problema estão surgindo iniciativas para liberar dados em um formato processável pela máquina, são os Dados Abertos [Colpaert et al. 2013]. Apesar do termo Dados Abertos ter sido concebido e ganhado força nos anos recentes, ele é um fenômeno inspirado em outros movimentos anteriores, como o Open Source [Weber 2004], que cresceu a partir de um interesse dos indivíduos, até chegar a produtos comercialmente viáveis. 
Os dados abertos têm se tornado um recurso importante no contexto educacional. Vários trabalhos estão sendo desenvolvidos para lidar com diferentes problemas educacionais a partir dos dados fornecidos pelo IDEB ${ }^{1}$. Alguns exemplos de utilização de dados abertos na educação são: apoiar a aprendizagem colaborativa [Fritzen et al. 2014]; diminuir a evasão [Rigo et al. 2012]; predição de desempenho de escolas [Adeodato et al. 2014].

Diante deste cenário, este trabalho apresenta uma revisão da literatura brasileira sobre trabalhos que utilizam dados abertos no contexto educacional publicados em eventos, periódicos, teses e dissertações. Destacaram-se as principais obras, mapeando-se os objetivos pedagógicos e as origens das publicações. A revisão teve o propósito de identificar e relatar as principais características de pesquisas na área no país.

Este trabalho está dividido nas seguintes seções: Na seção 2, são apresentados detalhes sobre dados abertos e dados abertos educacionais. A seção 3 explica a metodologia empregada na busca por trabalhos. Na seção 4 são descritos os resultados obtidos. Na seção 5 são discutidos trabalhos relevantes encontrados nesta revisão e possíveis linhas de pesquisa existentes nesta área. Por fim, na seção 6 são apresentadas considerações finais e trabalhos futuros.

\section{Dados Abertos}

Segundo a definição da Open Knowledge Foundation [Bauer and Kaltenböck 2011], os dados são abertos quando qualquer pessoa pode livremente usá-los, reutilizá-los e redistribuí-los, estando sujeito, no máximo, à exigência de creditar a sua autoria e compartilhar pela mesma licença. Isso geralmente é satisfeito pela publicação dos dados em formato aberto e sob uma licença aberta.

Embora o uso de dados abertos apresente vários benefícios, ainda existem discussões sobre legislações e motivações para liberar tais dados. É possível identificar duas razões principais para tal: (i) Transparência, onde se encontram principalmente os dados governamentais ou de empresas de capital aberto. Os dados abertos são importantes para que se possa acompanhar o que está sendo feito nessas organizações; e (ii) Inovação, a partir do momento que é facilitado o acesso dos computadores aos conteúdos, através de disponibilização de dados em formatos de fácil processamento pela máquina, há possibilidades para criação de sistemas relacionados a eles. Alem disto, é importante ressaltar os oito princípios que pautam dados abertos em geral [Isotani and Bittencourt 2015]:

- Completos: Dados são informações eletronicamente gravadas, incluindo, mas não se limitando a, documentos, bancos de dados, transcrições e gravações audiovisuais. Dados públicos são dados que não estão sujeitos a limitações válidas de privacidade, segurança ou controle de acesso, reguladas por estatutos.

- Primários: Os dados são publicados na forma coletada na fonte, com a mais fina granularidade possível, e não de forma agregada ou transformada.

- Atuais: Os dados são disponibilizados o quão rapidamente seja necessário para preservar o seu valor.

- Acessíveis: Os dados são disponibilizados para o público mais amplo possível e para os propósitos mais variados possíveis.

\footnotetext{
${ }^{1}$ http://dados.gov.br/
} 
VI Congresso Brasileiro de Informática na Educação (CBIE 2017)

Anais do XXVIII Simpósio Brasileiro de Informática na Educação (SBIE 2017)

- Processáveis por máquina: Os dados são razoavelmente estruturados para possibilitar o seu processamento automatizado.

- Acesso não discriminatório: Os dados estão disponíveis a todos, sem que seja necessária identificação ou registro.

- Formatos não proprietários: Os dados estão disponíveis em um formato sobre o qual nenhum ente tenha controle exclusivo.

- Livres de licenças: Os dados não estão sujeitos a regulações de direitos autorais, marcas, patentes ou segredo industrial. Restrições razoáveis de privacidade, segurança e controle de acesso podem ser permitidas na forma regulada por estatutos.

Os Dados Abertos Educacionais (DAE) [Alcantara et al. 2015], foco principal deste trabalho, se referem especificamente aos dados abertos vindos das instituições de ensino, como escolas, universidades, bibliotecas, entre outras. Estes podem ser de vários tipos como:

- Dados Administrativos: dados referenciais, como endereços, coordenadas em gps;

- Dados Internos: dados de staff, recursos disponíveis, orçamento;

- Dados Pedagógicos: dados do curso, grades curriculares, objetivos de aprendizagem;

- Dados de Usuários: estatísticas de aprendizagem, avaliações, dados de performance.

É importante fazer um diferenciação entre Dados Abertos Educacionais e Recursos Abertos Educacionais (RAC) [DUTRA and TAROUCO 2007]. Os RAC são materiais que podem ser usados ensino e pesquisa, tendo como principal foco ajudar professores a criar aula mais iterativas e ricas. Eles estão focados no conteúdo propriamente dito e não nas estatísticas das escolas e alunos, como é o caso dos DAE.

\section{Metodologia}

Para a realização desta revisão foram definidas algumas etapas, caracterizando um fluxo de atividades sequenciais seguindo o modelo proposto em [Peña-Ayala 2014]. Este fluxo adaptado é mostrado na Figura 1.

A etapa de seleção das fontes foi baseada principalmente em duas categorias: periódicos e conferências. Além disto, o trabalho também buscou referências de teses, dissertações e trabalhos de conclusão de curso. Em todas as categorias, foram buscados trabalhos publicados no Brasil e que tenham alguma relação com educação ou sistemas computacionais.

$\mathrm{Na}$ etapa de parâmetros de busca, foi definido que a pesquisa seria realizada em páginas de conferências e periódicos da área de Informática na Educação utilizados em [Rodrigues et al. 2014], no Portal Periódicos da CAPES e na ferramenta Google Scholar. Além disto, as palavras chaves utilizadas para as buscas foram: "Dados abertos educacionais", "Dados abertos + educação", "INEP", "IDEB"e "ENEM". As duas primeiras são termos mais genéricos; por outro lado as últimas representam os principais tipos de dados abertos utilizados em trabalhos de educação. Vale salientar que foram considerados os 
VI Congresso Brasileiro de Informática na Educação (CBIE 2017)

Anais do XXVIII Simpósio Brasileiro de Informática na Educação (SBIE 2017)

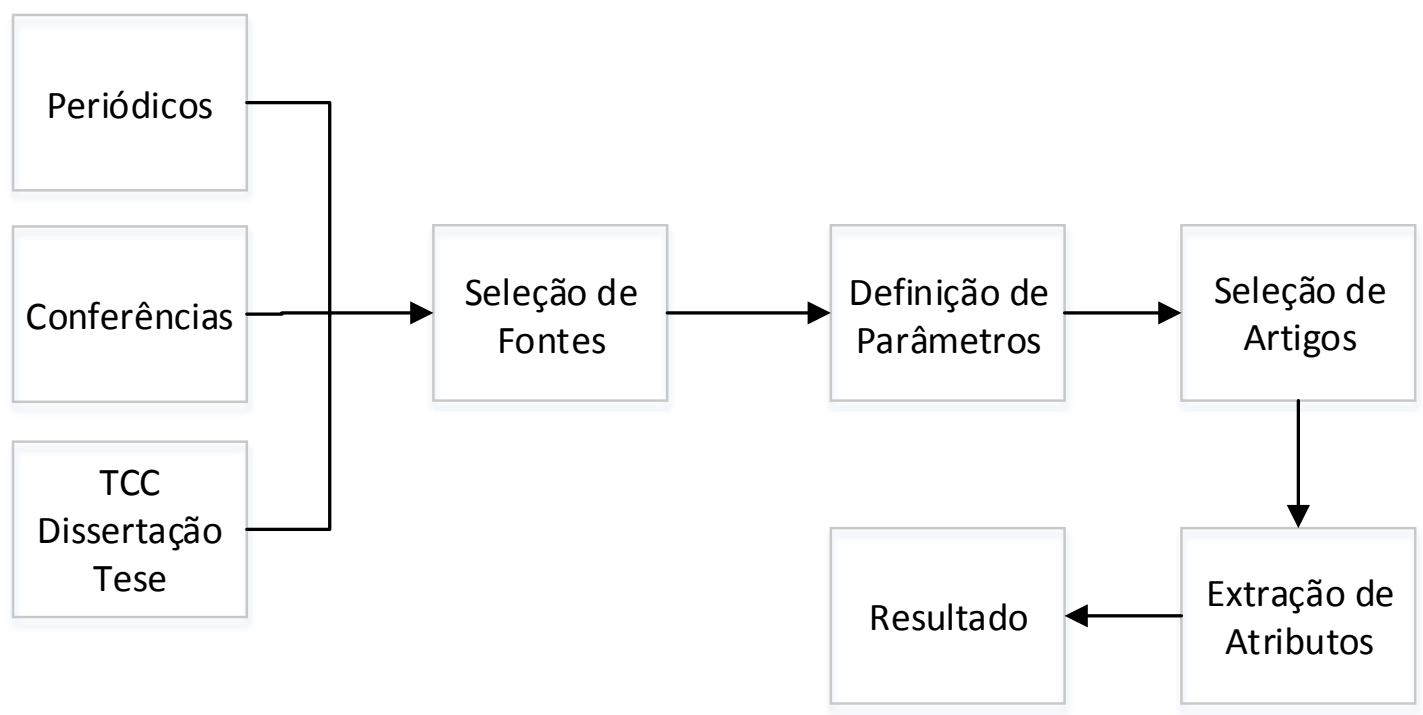

Figura 1. Fluxo de trabalho proposto

trabalhos publicados nos últimos 10 anos (2008-2017). A lista de trabalhos recuperados encontra-se no link: http://tiny.cc/DAERevisaoLiteratura.

$\mathrm{Na}$ terceira etapa foram selecionados aqueles artigos considerados relevantes. Foram excluídos artigos que: (i) estavam fora do período da pesquisa; (ii) não utilizam dados abertos educacionais, por exemplo artigos relacionados a recursos educacionais; e (iii) não utilizam dados abertos para aplicações educacionais.

\section{Resultados}

A busca inicial resultou em 47 artigos, e após a etapa de seleção 32 trabalhos foram considerados relevantes para o estudo. A Tabela 1 mostra a distribuição dos artigos em relação ao veículo no qual os artigos foram publicados. O pequeno número de artigos publicados em conferências mostra que a exploração de dados abertos educacionais na literatura brasileira ainda está em fase inicial.

Tabela 1. Distribuição dos Artigos por Categorias

\begin{tabular}{|c|c|}
\hline Categoria & Quantidade \\
\hline Conferências & 16 \\
\hline Periódicos & 7 \\
\hline Workshop & 5 \\
\hline Outros & 4 \\
\hline Total & $\mathbf{3 2}$ \\
\hline
\end{tabular}

Poucos trabalhos foram publicados, e menos ainda utilizam técnicas computacionais para extrair informações dos dados. Isso explica a grande concentração de artigos em eventos como Workshop de Informática na Escola (WEI) e Taller Internacional de Software Educativo (TISE) que aceitam mais análises qualitativas e estudos de caso. Como se 
VI Congresso Brasileiro de Informática na Educação (CBIE 2017)

Anais do XXVIII Simpósio Brasileiro de Informática na Educação (SBIE 2017)

pode ver na tabela 2, a conferência que teve mais artigos relevantes foi o Simpósio Brasileiro de Informática na Educação, enquanto o periódico com maior número de publicações foi a Revista Brasileira de Informática na Educação. Por fim, foram encontrados 5 trabalhos classificados como outros, estes incluem os TCCs e dissertações.

Tabela 2. Distribuição dos Artigos por Tipo de Fonte

\begin{tabular}{|rl|c|}
\hline Fonte & Quantidade \\
\hline Workshop & WIE & 5 \\
\hline & TISE & 3 \\
& SBSI & 1 \\
& SBIE & 7 \\
& DESAFIE & 2 \\
& SBSR & 1 \\
& SBSeg & 1 \\
& KDMiLe & 1 \\
\hline \multirow{5}{*}{ Periódicos } & RBIE & 2 \\
& iSys & 1 \\
& InCID & 1 \\
& TCU & 1 \\
& Revista Mediação & 1 \\
& Revista Percurso & 1 \\
\hline Outros & & 4 \\
\hline
\end{tabular}

Outro dado para confirmar que a área estudada neste trabalho ainda está começando a se desenvolver é a distribuição dos artigos ao longo dos anos, como mostra a Figura 2. Apesar da busca ter sido realizada para artigos a partir do ano de 2008, apenas em 2011 o primeiro artigo foi encontrado. Além disto, a quantidade de publicações no tema atingiu o maior número em 2015 com 11 artigos.

Os principais objetivos dos artigos selecionados, apresentados na Tabela 3, são:

- Mineração de dados aplicados a dados abertos educacionais: Dentro desse objetivo encontramos três tipos de artigos, sistemas para apoiar o gestor na tomada de decisão; predição de desempenho de escolas; e análise de algoritmos de aprendizagem de máquina focados para dados abertos educacionais.

- Análise qualitativa: Análise relativa a qualidade de dados abertos educacionais, possibilidades de aplicações e estudos de contextos escolares que se adequam à utilização dos mesmos.

- Teórico: Nesta categoria encontram-se artigos que detalham métodos e desafios sobre a utilização de dados abertos educacionais.

- Sistema para apoio a aprendizagem: Utilização de dados abertos para apoiar a aprendizagem colaborativa.

- Visualização dos dados: Aplicações que possibilitam uma melhor visualização dos dados abertos educacionais de uma determinada região.

Por fim, em junho de 2017 foi coletado no Google Scholar o número de citações de cada artigo, para estabelecer um ranking dos trabalhos referenciados na literatura (ver tabela 4). Esse resultado evidencia mais uma vez que esta área ainda é pouco explorada, 
VI Congresso Brasileiro de Informática na Educação (CBIE 2017)

Anais do XXVIII Simpósio Brasileiro de Informática na Educação (SBIE 2017)

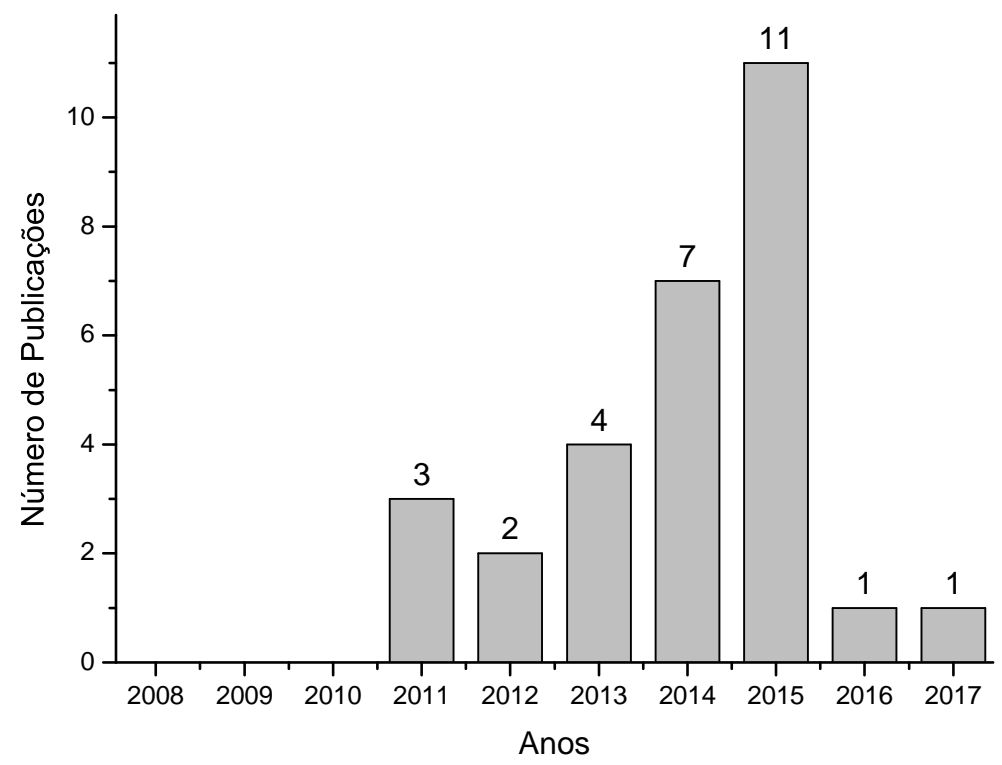

Figura 2. Número de publicações ao longo dos anos

Tabela 3. Objetivo Principal do Artigo

\begin{tabular}{|l|c|}
\hline Categoria & Quantidade \\
\hline Mineração de dados aplicados a dados abertos educacionais & 10 \\
\hline Análise qualitativa & 9 \\
\hline Teórico & 6 \\
\hline Sistema para apoio a aprendizagem & 3 \\
\hline Visualização dos dados & 3 \\
\hline
\end{tabular}

pois os principais trabalhos estão classificados como teóricos ou de análises qualitativas. O que mostra que ainda existe muito espaço para a utilização de técnicas computacionais para extrair informações de dados abertos educacionais.

Tabela 4. Trabalhos mais citados

\begin{tabular}{|l|c|}
\hline Título & N Citações $^{\mathbf{0}}$ \\
\hline $\begin{array}{l}\text { Mineração de Dados Educacionais: Oportunidades para o } \\
\text { Brasil [Baker et al. 2011] }\end{array}$ & 84 \\
\hline $\begin{array}{l}\text { Minerando Dados Educacionais com foco na evasão escolar: } \\
\text { oportunidades, desafios e necessidades [Rigo et al. 2012] }\end{array}$ & 43 \\
\hline $\begin{array}{l}\text { O uso das TIC na formação de professores de escolas que } \\
\text { obtiveram baixo IDEB [Coelho Neto et al. 2011] }\end{array}$ & 4 \\
\hline $\begin{array}{l}\text { Avaliando as competências escolares através da Prova Brasil } \\
\text { usando ferramenta web [Pinheiro et al. 2013] }\end{array}$ & 3 \\
\hline $\begin{array}{l}\text { Interação mútua e docência mediadora: Subsídios para avaliar a } \\
\text { aprendizagem na educação online [Koehler and Carvalho 2012] }\end{array}$ & 4 \\
\hline
\end{tabular}


VI Congresso Brasileiro de Informática na Educação (CBIE 2017)

Anais do XXVIII Simpósio Brasileiro de Informática na Educação (SBIE 2017)

\section{Discussão}

Com a grande disseminação dos dados abertos, especialmente os dados governamentais, a quantidade de aplicações para utilizá-lo vem crescendo cada vez mais. Na literatura brasileira, existem diversos sistemas que utilizam dados abertos para obter informações sobre política, saúde e economia. No contexto educacional, apesar de existirem muitas fontes de dados, as pesquisas ainda estão em estágio inicial de desenvolvimento.

De acordo com os resultados apresentados no trabalho proposto é possível perceber que existem poucos artigos relacionados à este tópico, porém também fica bem evidenciado o potencial de desenvolvimento e interesse principalmente pelo grande crescimento de publicações nos últimos anos.

Grande parte dos trabalhos encontrados na literatura são análises qualitativas sobre possíveis aplicações de mineração de dados educacionais [Baker et al. 2011]. Contudo, mesmo sendo estudos preliminares, os artigos apontam vários benefícios da aplicação destes dados. As principais aplicações estudadas são:

- Avaliação do ensino em vários níveis, desde escolas até estados [Pinheiro et al. 2013];

- Utilização de tecnologia na sala de aula [Generoso et al. 2013];

- Análise dos dados educacionais existentes [de Assis Rodrigues et al. 2015];

- Combate a evasão [Rigo et al. 2012].

Outro ponto importante de se destacar é a utilização de técnicas de mineração de dados aplicadas aos dados abertos educacionais. A partir de dados disponibilizados pelo INEP, diferentes trabalhos propõem a utilização de algoritmos de aprendizagem de máquina, como regressão logística e árvore de decisão, para extrair informações úteis para gestores de escolas [Adeodato et al. 2014, Ferreira 2015].

Com intuito de facilitar a visualização de dados abertos educacionais a plataforma CultivEduca [Carvalho et al. 2016] apresenta informações relativas a perfis de docentes em diferentes estados do Brasil. Além disto, os trabalhos [Guerra et al. 2014] e [da Silva et al. 2014] propõem que a visualização dos dados pode auxiliar gestores de cidades e estados a tomar decisões sobre investimentos em escolas. Os dados abertos também podem ser usados para auxiliar a aprendizagem através de sistemas de apoio à pesquisa e sistemas para auxiliar a aprendizagem colaborativa [Fritzen et al. 2014, de Sousa and da Silva 2015].

Por fim, é importante destacar trabalhos mais teóricos e relacionados a características das escolas, disponibilização de dados abertos e avaliação de algoritmos de aprendizagem de máquina. Santos et al. [Santos et al. 2014] discute que a infraestrutura das escolas impacta a qualidade do ensino das escolas apontando a eficiência trazida quando o governo investe na educação e a implicação no aprendizado dos alunos a partir da qualidade de ensino resultante da infraestrutura das escolas. O trabalho Dados abertos conectados para a educação [Bandeira et al. 2015] apresenta boas práticas de como se publicar dados abertos e detalha vantagens e limitações do cenário atual dos dados abertos educacionais. Em Comparação de algoritmos do aprendizado de máquina aplicados na mineração de dados educacionais [de Souza 2015] é apresenta a avaliação de algoritmos supervisionados aplicados a dados abertos educacionais. 
Além dos trabalhos científicos, outras iniciativas tem surgido no contexto de dados abertos educacionais. Em 2014, no Reino Unido, ocorreu um desafio chamado Education Open Data Challenge ${ }^{2}$. O desafio consistia em criar soluções que ajudassem pais e responsáveis a tomar decisões sobre seus filhos, expressando preferência por alguma escola, escolhendo um assunto ou alguma prioridade de aprendizagem, ou ainda praticar com a aprendizagem dos seus filhos. O vencedor foi a aplicação Skills Route, que foi projetada para mostrar opções de cursos e instituições de ensino de graduação disponíveis traçando parâmetros para projetar opções de carreira e até salários possíveis.

O Laboratório de Dados Abertos Brasil ${ }^{3}$ possui uma aplicação para Web denominada Educação Inteligente, onde são utilizados dados públicos, principalmente do INEP. É uma plataforma de captura, análise, manipulação e publicação dos dados governamentais sobre a educação, permitindo a busca por escola ou por estatísticas de região, estado ou cidade, possibilitando, por exemplo, apresentação dos dados à população e aos gestores públicos.

O QEdu ${ }^{4}$ é uma plataforma na web que agrega dados de avaliações e indicadores nacionais, como a Prova Brasil, o Censo Escolar, IDEB e ENEM. Através do cruzamento de informações, a ferramenta auxilia gestores, professores e outros interessados a fazerem melhores escolhas na educação. Para isso, além de disponibilizar os dados, trabalha em outras frentes: Blog, onde geram e discutem ideias; Academia, que explica o funcionamento e o propósito dos indicadores e do próprio QEdu; e Redes, que fornece uma síntese de dados para redes municipais e estaduais.

O Reduca $^{5}$ é a Rede Latino-Americana de Organizações da Sociedade Civil pela Educação. O Observatório Educativo do Reduca é uma plataforma que reúne indicadores e informações educacionais dos 14 países das organizações que compõem a rede, o que inclui o Brasil. Seu objetivo é dar visibilidade aos dados educacionais da região para que a sociedade monitore e avalie políticas públicas educativas e promova o intercâmbio de boas práticas, auxiliando gestores, pesquisadores e formuladores de políticas públicas a desenvolver soluções para os desafios educacionais. As fontes de dados do Observatório vem de organismos internacionais como a Unesco e a Comissão Econômica para a América Latina e o Caribe - Cepal, além de censos demográficos, pesquisas domiciliares e avaliações nacionais elaborados por instituições de cada país da região.

\section{Conclusões}

Este trabalho teve como objetivo apresentar a revisão da literatura brasileira sobre dados abertos educacionais nos últimos dez anos (2008-2017). Contudo, apenas a partir de 2011 foram encontrados artigos relacionados a área estudada. Foram extraídas diferentes características sobre os artigos selecionados que levaram a apresentar uma visão geral da pesquisa brasileira sobre o assunto.

Apesar da ampla abertura existente para obter dados abertos e da grande diversidade de tecnologias disponíveis para a utilização de tais dados, ainda há poucas iniciativas

\footnotetext{
${ }^{2}$ https://theodi.org/education-open-data-challenge-series

${ }^{3}$ http://dadosabertosbr.org

${ }^{4} \mathrm{http}: / / w w w . q e d u . o r g . b r$

${ }^{5}$ http://www.todospelaeducacao.org.br
} 
VI Congresso Brasileiro de Informática na Educação (CBIE 2017)

Anais do XXVIII Simpósio Brasileiro de Informática na Educação (SBIE 2017)

brasileiras no campo referente aos dados educacionais. Uma evidência disto é o fato de apenas 32 artigos terem sido considerados relevantes para os critérios de busca definidos.

Como sugestões de trabalhos futuros temos: (i) A divulgação do resultado desta coleta de dados com o objetivo de fomentar iniciativas que trabalhem com dados abertos educacionais; (ii) Realização de pesquisa da literatura em inglês que trata do tema; (iii) Propor aplicações para utilização dos dados educacionais.

\section{Referências}

Adeodato, P. J., Santos Filho, M. M., and Rodrigues, R. L. (2014). Predição de desempenho de escolas privadas usando o enem como indicador de qualidade escolar. In Simpósio Brasileiro de Informática na Educação, volume 25, page 891.

Alcantara, W., Bandeira, J., Barbosa, A., Lima, A., Ávila, T., Bittencourt, I., and Isotani, S. (2015). Desafios no uso de dados abertos conectados na educação brasileira. In DesafiE-4o Workshop de Desafios da Computação Aplicada à Educação. CSBC.

Baker, R., Isotani, S., and Carvalho, A. (2011). Mineraçao de dados educacionais: Oportunidades para o brasil. Brazilian Journal of Computers in Education, 19(02):03.

Bandeira, J., Ávila, T., Alcantara, W., Sobrinho, A., Bittencourt, I. I., and Isotani, S. (2015). Dados abertos conectados para a educação. Jornada de Atualização em Informática na Educação, 4(1):47-69.

Bauer, F. and Kaltenböck, M. (2011). Linked open data: The essentials. Edition mono/monochrom, Vienna.

Carvalho, M. J. S., Neves, B., and Melo, R. (2016). Plataforma cultiveduca. In Workshop do Congresso Brasileiro de Informática na Educação, volume 5, page 134.

Coelho Neto, J., Bernardelli, M., Pessoa, M., GUILHEM, S., Malucelli, A., and Reinehr, S. (2011). O uso das tic na formação de professores de escolas que obtiveram baixo ideb. Simpósio Brasileiro de Informática na Educação, pages 988-996.

Colpaert, P., Joye, S., Mechant, P., Mannens, E., and de Walle, R. (2013). The 5 stars of open data portals. In Conference on Methodologies, Technologies and Tools enabling e-Government 2013 Conference Proceedings.

da Silva, C. F., dos Santos, E. M. F., Chaves, M. C., Vaz, W., and Balaniuk, R. (2014). Dados abertos: uma estratégia para o aumento da transparência e modernização da gestão pública. Revista do TCU, (131):22-29.

de Assis Rodrigues, F., Sant'Ana, R. C. G., and Ferneda, E. (2015). Análise do processo de recuperação de conjuntos de dados em repositórios governamentais. InCID: Revista de Ciência da Informação e Documentação, 6(1):38-56.

de Sousa, R. and da Silva, L. É. P. (2015). Bravo sistema web de apoio à pesquisa em educação. In Workshop do Congresso Brasileiro de Informática na Educação, volume 4, page 105 .

de Souza, M. N. V. (2015). Comparação de Algoritmos do Aprendizado de Máquina Aplicados na Mineração de Dados Educacionais. Tcc, Universidade Federal Rural de Pernambuco. 
VI Congresso Brasileiro de Informática na Educação (CBIE 2017)

Anais do XXVIII Simpósio Brasileiro de Informática na Educação (SBIE 2017)

DUTRA, R. L. d. S. and TAROUCO, L. M. R. (2007). Recursos educacionais abertos (open educational resources). Novas Tecnologias na Educação, 5(1).

Ferreira, G. (2015). Investigação acerca dos fatores determinantes para a conclusão do ensino fundamental utilizando mineração de dados educacionais no censo escolar da educação básica do inep 2014. In Workshop do Congresso Brasileiro de Informática na Educação, volume 4, page 1034.

Fritzen, E., Siqueira, S. W. M., and de Vasconcelos Andrade, L. C. (2014). Busca contextualizada enriquecida com dados abertos para apoiar a aprendizagem colaborativa em redes sociais. Revista Brasileira de Informática na Educação, 21(03):25.

Generoso, A. A. P., Neto, J. C., Reinehr, S., and Malucelli, A. (2013). Abordagem qualitativa do uso das tdic na educação básica. In Workshop de Informática na Escola, volume 1, page 230 .

Guerra, P. C., Nakamura, R. Y. M., Hruschka, E. R., et al. (2014). Estimativa de demanda potencial de matrículas em ensino superior usando dados públicos e múltiplos modelos de regressão. In Symposium on Knowledge Discovery, Mining and Learning, 2th. Sociedade Brasileira de Computação-SBC.

Isotani, S. and Bittencourt, I. I. (2015). Dados Abertos Conectados. Novatec Editora.

Koehler, C. and Carvalho, M. J. S. (2012). Interação mútua e docência mediadora: subsídios para avaliar a aprendizagem na educação online. In Proceedings TISE-2012XVII Congreso Internacional de Informática Educativa. pg, pages 367-368.

Peña-Ayala, A. (2014). Educational data mining: A survey and a data mining-based analysis of recent works. Expert systems with applications, 41(4):1432-1462.

Pinheiro, R. G. P., Elia, M., and Sampaio, F. F. (2013). Avaliando as competências escolares através da prova brasil usando ferramenta web. In Workshop de Informática na Escola, volume 1, page 60.

Rigo, S. J., Cazella, S. C., and Cambruzzi, W. (2012). Minerando dados educacionais com foco na evasão escolar: oportunidades, desafios e necessidades. In Workshop de Desafios da Computação Aplicada à Educação, pages 168-177.

Rodrigues, R. L., Ramos, J. L. C., Silva, J. C. S., and Gomes, A. S. (2014). A literatura brasileira sobre mineração de dados educacionais. In Workshops do Congresso Brasileiro de Informática na Educação, volume 3, page 621.

Santos, M. S., Claro, D. B., de Souza Oliveira Filho, A. M., Paoli, A. R., Barbosa, C. D., Almeida, E. V., de Araújo Carvalho, G. D., de Jesus Santana, H., Bulcão, L. A., Marotta, L. L., et al. (2014). Análise das infraestruturas do censo escolar 2011: uma proposta da disciplina de tópicos em banco de dados. In Simpósio Brasileiro de Informática na Educação, volume 25, page 124.

Weber, S. (2004). The success of open source, volume 897. Cambridge Univ Press.

Wood, D., Zaidman, M., Ruth, L., and Hausenblas, M. (2013). Linked Data: Structured data on the Web. New York: Manning Publications Co., 1th edition. 